\title{
月表光照区简单陨坑的有效太阳辐照度和温度的 数值模拟
}

\author{
甘红 $1,2 *$ ，李雄耀 3,4 ，魏广飞 $2,3,4$ \\ 1. 贵州理工学院分析测试中心, 贵阳 550003; \\ 2. 澳门科技大学月球与行星科学国家重点实验室, 澳门; \\ 3. 中国科学院地球化学研究所月球与行星科学研究中心, 贵阳 550081; \\ 4. 中国科学院比较行星学卓越创新中心, 合肥 230026 \\ *联系人, E-mail: ganhong06@gmail.com
}

收稿日期: 2018-10-12; 接受日期: 2019-01-08; 网络出版日期: 2019-04-24

国家自然科学基金(编号: 41572037, 41803052)、中国科学院B类先导科技专项(编号: XDPB11)、澳门科学技术发展基金(编号: 119/2017/A3)和贵州理 工学院高层次人才科研启动项目(编号: XJGC20181290)资助项目

摘要 太阳辐射是影响月表热环境的主要因素, 前人的研究大多忽略月表地形的影响或仅考虑大尺度的 地形起伏. 本文以Banting 简单陨坑 $\left(16.4^{\circ} \mathrm{E}, 26.6^{\circ} \mathrm{N}\right)$ 为例, 首先基于月球勘探轨道器 $(\mathrm{LRO})$ 搭载的月球轨道器 激光测高仪获取的地形数据构建了该陨坑的三维数值模型, 计算了不同地方时条件下的有效太阳辐照度分 布. 其次, 本文以具有Banting陨坑相同深径比的陨坑为研究对象, 计算了其在月表任意位置和时刻的光照面 积占陨坑面积的分布(光照面积占比). 结果表明, 在 $48.2^{\circ} \mathrm{N} / \mathrm{S}$ 和当地时间 08:48-15:12的时空范围内, 陨坑的光 照面积占比达 $100 \%$ 。最后, 根据月表热辐射平衡模型计算了Banting陨坑在白天不同时刻的温度分布. 通过 与LRO月球辐射实验仪的测量温度对比, 二者表现出较好的一致性, 验证了数值模拟的可靠性.

关键词月球, 简单陨坑, 太阳辐照度, 温度, 数值模拟

PACS: 95.30.Jx, 95.85.Hp, 96.20.-n, 96.35.Gt

\section{1 引言}

太阳辐射是驱动月表温度变化的主要热源, 也 是研究月表热环境考虑的主要因素. 对月表温度的 测量一般有直接测量、利用样品热物理性质推测、 遥感探测、地基观测、物理模型等方法 ${ }^{[1]}$. 由于月 表地形起伏尤其是密集分布的陨坑使得月表热环境 变得十分复杂, 通常用有效太阳辐照度和太阳光照
率(某一位置的光照时间占总时长的百分比)来表示 其光照特征. 其中有效太阳辐照度控制着月表的温 度, 而光照率则影响月面活动的时间, 它们是月球着 陆点和未来基地选址的重要影响因素.

有效太阳辐照度是影响月表温度分布的重要 因素. $\mathrm{Li}$ 等人 ${ }^{[2] 、 F e n g}$ 等人 ${ }^{[3]}$ 根据月表有效太阳辐 照度与日月距离、太阳辐射入射角的关系, 建立了 月表有效太阳辐照度的实时模型. 刘书豪 ${ }^{[4]}$ 提出

引用格式: 甘红, 李雄耀, 魏广飞. 月表光照区简单陨坑的有效太阳辐照度和温度的数值模拟. 中国科学: 物理学 力学 天文学, 2019, 49: 069601 Gan H, Li X Y, Wei G F. Numerical simulation of effective solar irradiance and temperatures at simple crater of lunar dayside (in Chinese). Sci Sin-Phys Mech Astron, 2019, 49: 069601, doi: 10.1360/SSPMA2018-00348 
了一种基于应用计算几何的月面任意位置的太阳 辐照度仿真模型. 以上模型均是基于光滑球面的 假设, 未考虑月表真实地形如陨坑的影响. Meng等 人 ${ }^{[5]}$ 通过建立经纬向坡度的太阳辐射模型以探讨地 形对月面温度分布的影响, 但没有考虑太阳辐照度 随时间的变化以及坡面的二次反射和热辐射等情 况. 张吉栋等人 ${ }^{[6]}$ 基于月球行星历表和月球勘探轨 道器(LRO)搭载的月球轨道器激光测高仪(LOLA)数 据, 根据日-地-月几何关系建立了月表光照模型, 分 析了Aristarchus高原的光照特性, 但缺乏对月表热 环境的研究. 此后张吉栋等人 ${ }^{[7]}$ 改进了月球光照模 型, 充分考虑坡度条件以及日月距离对月表太阳辐 射的影响, 计算了冯.卡门陨坑2018年接受的太阳辐 射能量, 但该研究仍没有考虑坑壁的二次反射和热 辐射作用.

目前, 对月表光照条件和热环境的研究更多地 集中在两极地区. 主要是由于月球两极永久阴影 区分布有一定量的水冰 ${ }^{[8]}$, 是未来月球水资源探测 和开采的重要区域. 永久阴影区的分布与月表温度 的分布密切相关, 其主要受到地形特征的影响 [9]. Vasavada ${ }^{[10]}$ 采用太阳辐射和热辐射多次散射模型, 计算了月球和水星极区简单陨坑、平底陨坑的最 大和平均温度分布, 但没有详细讨论不同地方时 以及纬度效应对陨坑内温度的影响. 随着高精度 地形数据的获取, Noda等人 ${ }^{[11]} 、$ Bussey等人 ${ }^{[12]}$ 根 据KAGUYA卫星获取的激光高度计数据和月球行 星历表计算了月球极区的光照率. Paige等人 ${ }^{[13]}$ 同样 根据KAGUYA激光高度计数据采用光线追踪法计 算了月球南极地区年均温度分布, 探讨了潜在水冰 的埋藏深度. Mazarico等人 ${ }^{[14]}$ 基于LOLA数据采用 最大高度角法计算了极区的光照条件并分析了散射 光对永久阴影坑挥发物的影响. 郝卫峰等人 ${ }^{[15]}$ 基于 嫦娥一号地形数据同样采用地形最大高度角法分析 了月球极区的光照条件, 发现南极比北极地区有更 大面积的永久阴影区, 且主要分布在大而深的撞击 坑. 他们仅给出了极区光照率的分布或年均温度分 布特征, 并没有讨论陨坑在不同空间条件下的有效 太阳辐照度和热环境瞬时变化特征.

地形的遮挡使得陨坑内外接受的太阳辐射能量 差异非常大. 早期Pettit等人 ${ }^{[16] 、 S i n t o n ~}{ }^{[17]}$ 基于地基
观测发现月球正面的热辐射强度从中心向边缘并 不是按照理想的余弦曲线变化, 即光滑朗伯体球面, 而是在边缘地区出现明显的亮温增强现象. Buhl等 人 ${ }^{[18]}$ 通过建立陨坑模型考虑了坑壁的二次辐射、 局部入射角、发射角和遮蔽效应对亮温的影响, 从 而解释了月球正面边缘亮温增强的观测现象. 当讨 论复杂地形如陨坑的热环境时, 往往需要考虑陨坑 的自身遮蔽效应、纬度效应以及二次辐射等因素的 影响 ${ }^{[9,19]}$. 由于陨坑特殊的形状, 其接受和被遮蔽的 太阳辐射是随时间不断变化的, 即太阳辐射到达坑 壁, 部分被反射其余被吸收, 吸收的能量则再次以热 辐射的形式发射出去. 这些过程又与陨坑所在的纬 度位置和地方时密切相关, 针对这一复杂过程本文 对地方时、纬度效应对陨坑热辐射环境的影响展开 讨论.

本文以位于澄海中央附近的Banting陨坑为研 究对象, 首先介绍了构建该陨坑的数学模型、有 效太阳辐照度和温度的方法. 其次, 给出了不同地 方时条件下Banting陨坑的有效太阳辐照度和表面 温度分布的数值模拟结果, 并进一步讨论了纬度 效应对该类型陨坑接受的有效太阳辐射面积的影 响. 最后, 基于LRO探测任务获取的热红外数据分析 了Banting陨坑不同地方时的温度分布, 对比验证了 数值模拟结果的可靠性.

\section{2 数据和研究方法}

\subsection{Diviner数据}

月球勘探轨道飞行器(LRO)发射于2009年6月 18 日, 其搭载的月球辐射计实验仪(Diviner Lunar Radiometer Experiment, Diviner), 是第一个系统测量 月表热环境和矿物成分的探测器 ${ }^{[20]}$, 也是目前唯 一能提供全月热红外波段测量数据的仪器. Diviner 辐射计的科学目标之一是系统地探测月表热 环境绘制全月温度图, 共包含9个观测通道且分布 于两个波段范围. 其中第 1 和 2 通道波段范围为 $0.35-$ $2.8 \mu \mathrm{m}$, 用于测量月表反射的太阳辐射; 第3-9 通道 范围为7.55-400 $\mu \mathrm{m}$, 用于测量月表红外辐射能量. 每个观测通道都含有 21 个水平的线性探测器单元阵 
列并采用连续推扫式测量 ${ }^{[20]}$. LRO进入到科学探测 任务阶段后的在轨运行高度为 $50 \mathrm{~km}$ 时, Diviner辐 射计的顺轨和交轨方向空间分辨率达 $320 \mathrm{~m} \times 160 \mathrm{~m}$, 刈幅宽度为 $3.4 \mathrm{~km}$. 在轨运行 8 年多, 已获得月表任 意位置的昼夜温度变化数据 ${ }^{[21,22]}$.

Bandfield等人 ${ }^{[23]}$ 在利用Diviner数据计算月 表温度和石块丰度等信息时发现，通道3至 通道5的月球夜晚低温时没有足够的信噪比, 不适合分析温度起伏剧烈的月表昼夜温度; 在Diviner的定标观测中, 通道9的亮温数据存 在严重漂移. 由于通道7 $(25-41 \mu \mathrm{m})$ 在整个黑 体温度范围内都有很高的信噪比，Vasavada等 人 ${ }^{[24]}$ 则仅根据通道7 亮温数据探讨了赤道地区 月壤的热物性. 本文同样采用覆盖Banting陨坑 的Diviner第7通道亮温数据(http://oderest.rsl.wustl. edu/GDSWeb/GDSDLRERDR.html), 以探讨不同时 刻的热环境特征.

\section{2 简单陨坑的三维模拟一以Banting陨坑为例}

月表陨坑不仅数量多,而且存在多种构造类型. 有复杂的大型多环撞击盆地, 如东海; 有包含中央 峰的撞击盆地, 如第谷撞击坑 ${ }^{[25,26]}$; 还有呈碗形的 简单陨坑, 通常直径小于 $15 \mathrm{~km}^{[27]}$. 尤其对于陨坑密 集分布的高地, 陨坑的叠覆使得月表地形变得更加 复杂. 相对于大型撞击坑, 简单陨坑的地形特征较 为简单, 在进行数值模拟时可以通过较少的参数对 其特征进行限定. 而且在中低纬度区域太阳辐照度 更大, 更适合陨坑探测中的太阳能利用. 为避免周 围起伏地形或陨坑叠覆等地形对陨坑热环境的影 响, 故本文选取位于澄海中央附近中心坐标 $\left(16.4^{\circ} \mathrm{E}\right.$, $26.6^{\circ} \mathrm{N}$ )的Banting 陨坑为研究对象, 其光学影像如 图1(a)所示. 可以看出, Banting陨坑具有典型的简单 陨坑特征且表面较为光滑, 直径约为 $6.1 \mathrm{~km}$, 坑底位 置相对平坦, 可能由坑壁碎屑滑落堆积导致. 但这对 本文的数值模拟没有影响.

根据LOLA数据可以得到Banting陨坑的高程分 布, 如图1(b)所示. 可以看到, Banting陨坑深度约 $1.1 \mathrm{~km}$, 坑缘周围地形平坦且坑壁坡度连续没有明 显陡坡. 对于此类陨坑一般可通过截取球面的部分
曲面, 再由曲面向周围延伸平面来进行模拟. 陨坑的 大小由坑深 $(H)$ 和坑径 $(D)$ 描述,一般用二者比值表 示, 即深径比 $\Delta=H / D$. 研究表明, 月球上 $D<15 \mathrm{~km}$ 的简单陨坑中深径比一般为 $1 / 6<\Delta<1 / 5$, 且均可用 截取的球面作为近似 ${ }^{[28,29]}$. 相比Banting陨坑直径, 月表的球面曲率对陨坑的影响可以忽略不计. 本文 以 $x, y$ 轴方向确定的 $x-y$ 平面表示月面, 陨坑的深度 为 $z$ 轴方向建立 $x-y-z$ 三维笛卡尔坐标系. 则陨坑任意 位置的高程 $(z)$ 可表示为 ${ }^{[30]}$

$z=R-H \pm \sqrt{R-x^{2}-y^{2}}$,

其中, $R=\left(D^{2} / 4+H^{2}\right) /(2 H)$ 为与月球表面相交的 大球面的半径. 根据式(1)可建立经向 $(x)$ 和纬向 $(y)$ 的网格坐标, 并构建Banting陨坑的三维地形, 如 图1(c)所示, 其中 $\Delta=H / D=1.1 / 6.1 \approx 0.18$. 注意, 在给定 $\Delta$ 值并忽略月球表面曲率的条件下, 坑径和 坑深的实际取值对本文模拟结果没有任何影响. 对 比图1(b)可以看出, 数值模拟陨坑可以很好地代表 真实陨坑的地形特征. 因此, 本文将基于模拟陨坑 进一步探讨陨坑的太阳辐照度和热环境特征.

\section{3 有效太阳辐照度及温度}

为计算有效太阳辐照度, 我们将数值模拟的 陨坑划分为 $N \times N$ 个等面积的网格, 在 $x, y$ 方向的单 元网格分别用 $i, j(i, j=1, \cdots, N)$ 表示. 为计算陨坑 各个位置的坡度, 这里需要将由 4 个网格点连接的 网格单元进一步划分为两个三角网格面 ${ }^{[30]}$, 则得 到 $2 \times N \times N$ 个三角网格面. 假设月表地形对太阳辐 射的散射为朗伯散射, 太阳辐射到达坑壁的能量即 有效太阳辐照度取决于日月距离、局部太阳入射 角. 由此可以计算陨坑内各个网格面之间的太阳辐 射和热红外辐射能量的散射.

对于任一天体如月球, 水平表面某一位置的太 阳高度角 $(\beta)$ 可用纬度 $(\lambda)$ 、太阳赤纬 $(\delta)$ 、时角 $(h)$ 表 示为 ${ }^{[31]}$

$\sin \beta=\cos \lambda \cos \delta \cos h+\sin \lambda \sin \delta$,

当 $\sin \beta<0$ 时, 假设太阳位于该无限延伸水平面以 下. 针对地形起伏的月表, 需要进一步考虑斜坡面 

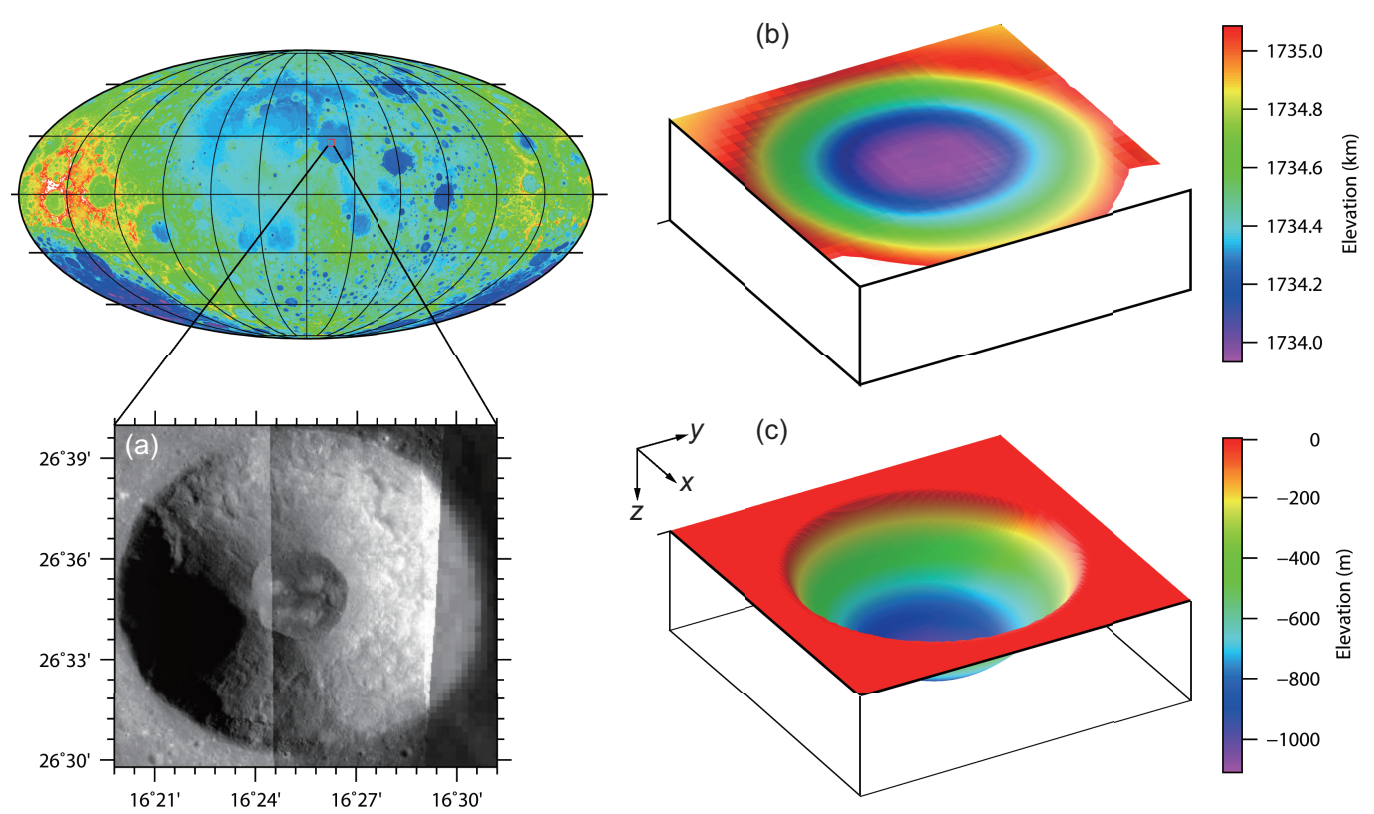

图 1 (网络版彩图)Banting陨坑的光学影像(a)、三维数字高程(b)、三维数值模型(c). 注: 光学影像数据来源于LRO 广 角相机(http://ode.rsl.wustl.edu/moon/indexMapSearch.aspx), 高程数据来源于LRO LOLA (http://oderest.rsl.wustl.edu/GDSWeb/ GDSLOLARDR.html)

Figure 1 (Color online) (a) Optical image, (b) three-dimensional topographic map and (c) three-dimensional numerical model of Banting crater. Note: the optical data are obtained by LRO Wide Angle Camera (http://ode.rsl.wustl.edu/moon/indexMapSearch.aspx), and the elevation data are obtained by LRO LOLA (http://oderest.rsl.wustl.edu/GDSWeb/GDSLOLARDR.html).

上的局部太阳高度角 $(\theta)$. 对于任意斜坡面上的太阳 高度角可表示为 ${ }^{[31]}$

$\sin \theta=\cos \alpha \sin \beta-\sin \alpha \cos \beta \cos (\Delta \alpha)$,

其中, $\Delta \alpha$ 是太阳方位角 $(\alpha)$ 和地形坡度的差值. 当 $\sin \theta<0$ 时, 假设太阳位于以该局部坡面无限延伸的 平面以下，即坡面的自身遮蔽作用. 假设入射到月 表的太阳辐射为平行光, 根据式(2)和(3)可知, 月表 局部地形的太阳高度角主要受到 $\lambda$ 和 $h$ 的影响. 对于 陨坑内存在的光的散射和坑壁热辐射作用, 任意位 置则满足以下能量守恒

$(1-A)\left(J+Q_{\mathrm{ref}}\right)+\varepsilon K+k \frac{\partial T}{\partial z}=F$,

其中, 左边第一项表示太阳直接辐射和坑壁二次反 射贡献的能量, 第二项表示陨坑内漫反射场即热红 外辐射的贡献, 第三项表示月壤次表层向上传导贡 献的能量, 相比太阳辐射, 内部热流对表面温度的 影响可以忽略. 此外阿波罗样品分析表明, 月壤表 层(约 $2 \mathrm{~cm}$ 厚的月尘层)的热导率极低, 因此对于瞬
时平衡可忽略此项的贡献即 $k \approx 0$. 右边第一项表示 月表向外空间辐射的能量, 即 $F=\varepsilon \sigma T^{4}$. 其中 $J$ 为 太阳的直接辐射, $Q_{\mathrm{ref}}$ 为周围坑壁反射的太阳辐射, $A$ 为表面反照率(这里假设各个位置反照率相同). $\varepsilon$ 为表面热发射率, $\sigma$ 为Stephan-Boltzmann常数.

对于太阳的直接辐射, 网格面 $i$ 接受到的能量可 表示为 [30]

$J_{i}=S \sin \theta \frac{v_{i}}{R_{\mathrm{m}}^{2}}$,

其中, $S$ 为太阳常数, $R_{\mathrm{m}}$ 为月球到太阳距离 $(\mathrm{Au}), v_{i}$ 为 可见度参数, $v_{i}=1$ 或 0 . 即当网格面受到太阳辐射时 取值为 1 , 当位于阴影区时取值为 0 . 而陨坑内的漫 辐射场主要为坑壁对太阳辐射的二次反射、坑壁的 热红外辐射, 其分别表示为 ${ }^{[30]}$

$$
\begin{gathered}
Q_{\text {ref }_{i}}=\sum_{j \neq i}^{N} \alpha_{j i} A_{j} J_{j}, \\
K_{i}=\varepsilon \sigma \sum_{j \neq i}^{N} \alpha_{j i} T_{j}^{4},
\end{gathered}
$$


其中, $\alpha_{j i}$ 为视场系数, 表示陨坑内从单元面 $j$ 辐射到 单元面 $i$ 的能量比例, $A_{j}$ 为单元面 $j$ 的反照率. 实际上 由于无法准确获得各个单元面的反照率 $\left(A_{j}\right)$ 也为了 简化计算, 本文中假设 $A=A_{j}(j=1, \cdots, N)$, 即坑壁 所有单元面的反照率相同. $\alpha_{j i}$ 可通过计算视角系数 得到 $[10,19]$

$\alpha_{j i}=\frac{\cos \theta_{j} \cos \theta_{i}}{\pi d_{j i}^{2}} d S_{j}$,

其中, $\theta_{j}$ 和 $\theta_{i}$ 分别为网格面 $j, i$ 的法线与二者中心连 线的夹角, $d_{j i}$ 为两个单元面中心的距离, $d S_{j}$ 为 $j$ 网 格面的面积. 根据以上辐射平衡模型, 可以计算陨 坑内的漫辐射场进而得到温度的分布. 通过计算 发现, 坑壁表面反照率 $(A)$ 和热发射率 $(\varepsilon)$ 是影响温 度分布的重要因素. 需要注意的是, 影响反照率和 热发射率的因素有很多, 如表面粗粘度、岩块含量 及分布等. Bandfield等人 ${ }^{[23]}$, Vasavada等人 ${ }^{[24]}$ 对这 些因素进行了详细的讨论. 本文假设坑壁表面光滑 且月壤表层物性均一, 并采用经验值作为参考 $A=$ $0.127, \varepsilon=0.95$. 日月距离 $R_{\mathrm{m}} \approx 1 \mathrm{AU}$, 太阳常数 $S=$ $1366 \mathrm{~W} \mathrm{~m}^{-2}$.

\section{3 结果及讨论}

\section{1 有效太阳辐照度}

对于地理坐标确定的Banting陨坑, 这里仅考 虑地方时变化对有效太阳辐照度 $\left(E_{\mathrm{eff}}\right)$ 的影响. 如 图2所示, Banting陨坑的有效太阳辐照度随地方时 变化表现出显著的差异, 且在不同时间陨坑的自 身遮蔽效应差异很大. 从背光面坑壁到向阳面坑 壁, 太阳辐照度逐渐增加并呈平缓的渐变特征. 具 体来看, 在黎明(图2(a))和傍晚(图2(g))时刻坑内的 遮蔽效应最明显, 大约 $24.8 \%$ 的坑壁被自身遮蔽, 而 有效太阳辐照度均在 $1024.5 \mathrm{~W} \mathrm{~m}^{-2}$ 以下. 在地方时 为10:00(图2(b))和16:00(图2(f))时, 陨坑则接受更多 的太阳辐射, 仅 7.9\%的坑壁被自身遮蔽, 且光照区 太阳辐照度普遍增加, 最高达 $1271.7 \mathrm{~W} \mathrm{~m}^{-2}$. 在靠 近正午时刻(图2(c)和(e))坑壁已不存在自身遮蔽效 应, 只是有效太阳辐照度分布表现出明显的不均 匀性, 在202.2-1366 W m ${ }^{-2}$ 范围变化. 到了正午时 刻(图2(d))背光面有效太阳辐照度继续增大且分布 更均匀, 但背光面还是明显受到坡度的影响, 最低 约 $505.4 \mathrm{~W} \mathrm{~m}^{-2}$. 值得一提的是, Banting陨坑自身遮 蔽效应消失和开始的时间并不是在图2(c)和(e) 所示 的10:00和14:00时刻, 具体时间下文将详细讨论. 此 外, 坑缘周围水平区域的太阳辐射率则与时间表现 出很好的相关性. 越接近正午时刻有效太阳辐照度 越大, 即接受的太阳辐射能量也就越多. 二者对比 恰恰可以说明陨坑是影响月表太阳辐照度分布非常 重要的因素.

除了地方时影响陨坑太阳辐射率分布外, 纬度 效应也是另一个很重要的因素. 纬度效应改变了坑 壁局部太阳高度角, 使有效太阳辐照度分布更加复 杂. 图3展示了具有Banting陨坑特征(即具有相同深 径比)的陨坑在月表任意时刻、任意位置的光照区 面积占陨坑总面积的百分比分布(以下简称光照面 积占比). 可见在 $48.2^{\circ} \mathrm{N} / \mathrm{S}$ 范围且当地时间在 $08: 48-$ 15:12之间(由实心点围成的圆圈内)的陨坑, 光照面 积占比达到 $100 \%$, 即在此范围内陨坑没有自身遮蔽 效应. 相反, 越靠近黎明/傍晚时刻或两极位置陨坑 的遮蔽效应越明显, 光照面积占比最低降到 $70 \%$ 以 下. 由此可知, 在未来月球陨坑的探测任务中选择 在此范围内开展可确保太阳辐射能源的持续供应. 当然, 具有不同深径比的简单陨坑的光照面积占比 会有变化, 根据本文模型均可计算得到. 鉴于大多 数简单陨坑的深径比在 $1 / 6-1 / 5$ 之间, 因此本文的计 算结果仍具有一定的代表性.

为进一步对比分析Banting陨坑的太阳光 照特征, 本文分别在图3(a)中选取了位于不同 纬度的4条剖面, 即赤道, $26.6^{\circ} \mathrm{N}, 60^{\circ} \mathrm{N}$ 和 $85^{\circ} \mathrm{N}$, 其中 $26.6^{\circ} \mathrm{N}$ 为Banting陨坑中心所在的纬度, 如 图3(b)所示. 显然, 赤道和 $26.6^{\circ} \mathrm{N}$ 位置陨坑的光 照条件十分接近. 光照面积占比达到 $100 \%$ 的当 地时间范围分别为(08:48-15:12)和(09:18-14:42). 而 $60^{\circ} \mathrm{N}$ 和 $85^{\circ} \mathrm{N}$ 位置的陨坑始终存在遮蔽区域, 且 高纬度地区遮蔽效应更显著. 从图3还可以发现, 所 有与圆圈相交的纬度剖面均存在 $100 \%$ 光照占比, 而 被圆弧截取的线段则是其光照时长. 没有与圆圈相 交的纬度剖面则在白天任何时刻均存在遮蔽区域, 且距离圆圈越远遮蔽面积越大, 遮蔽时间越长. 

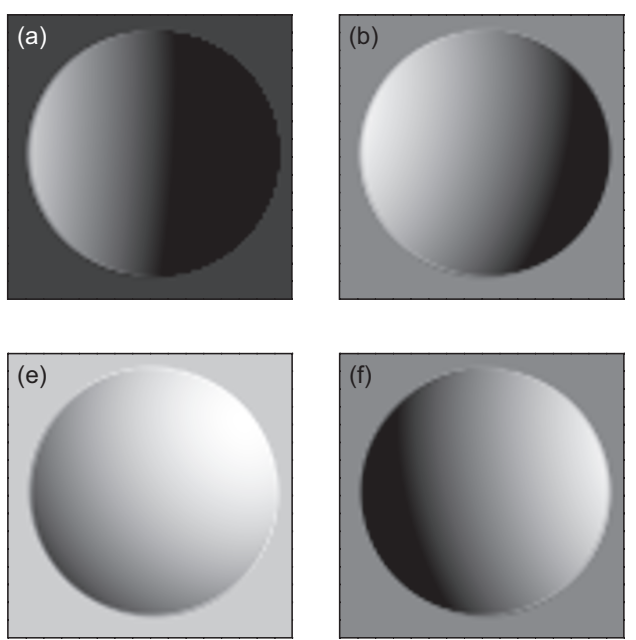
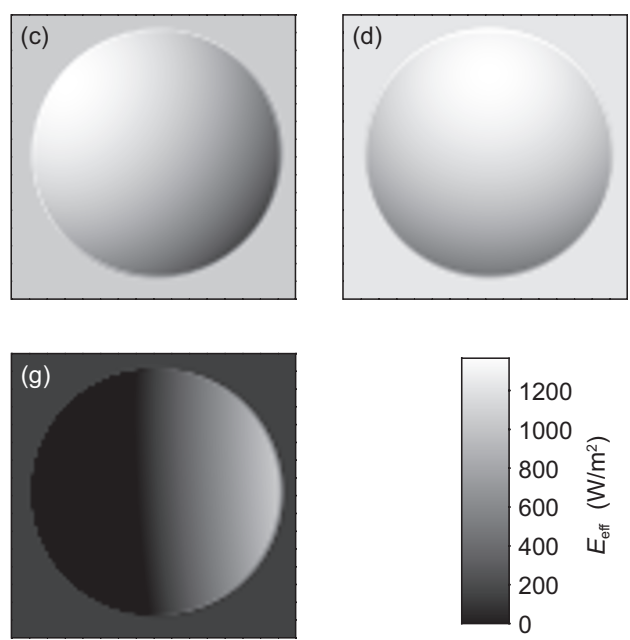

图 2 不同地方时条件下Banting陨坑 $\left(16.4^{\circ} \mathrm{E}, 26.6^{\circ} \mathrm{N}\right)$ 的有效太阳辐照度分布. (a)- $(\mathrm{g})$ 对应的地方时分别为 06:30, 08:00, 10:00, $12: 00,14: 00,16: 00,17: 30$. 注: 当 $E_{\mathrm{eff}}=0$ 时, 表示该位置完全被遮蔽; 为 $E_{\mathrm{eff}}=1366 \mathrm{~W} \mathrm{~m}^{-2}$ 时, 表示太阳直射该位置

Figure 2 Distribution of solar radiation rate of Banting crater $\left(16.4^{\circ} \mathrm{E}, 26.6^{\circ} \mathrm{N}\right)$ at different local times. (a) $-(\mathrm{g})$ correspond to the local time $06: 30$, 08:00, 10:00, 12:00, 14:00, 16:00, 17:30, respectively. Note: When $E_{\text {eff }}=0$, it is self-shaded. When it increases to $E_{\text {eff }}=1366 \mathrm{~W} \mathrm{~m}^{-2}$, it is illuminated vertically.
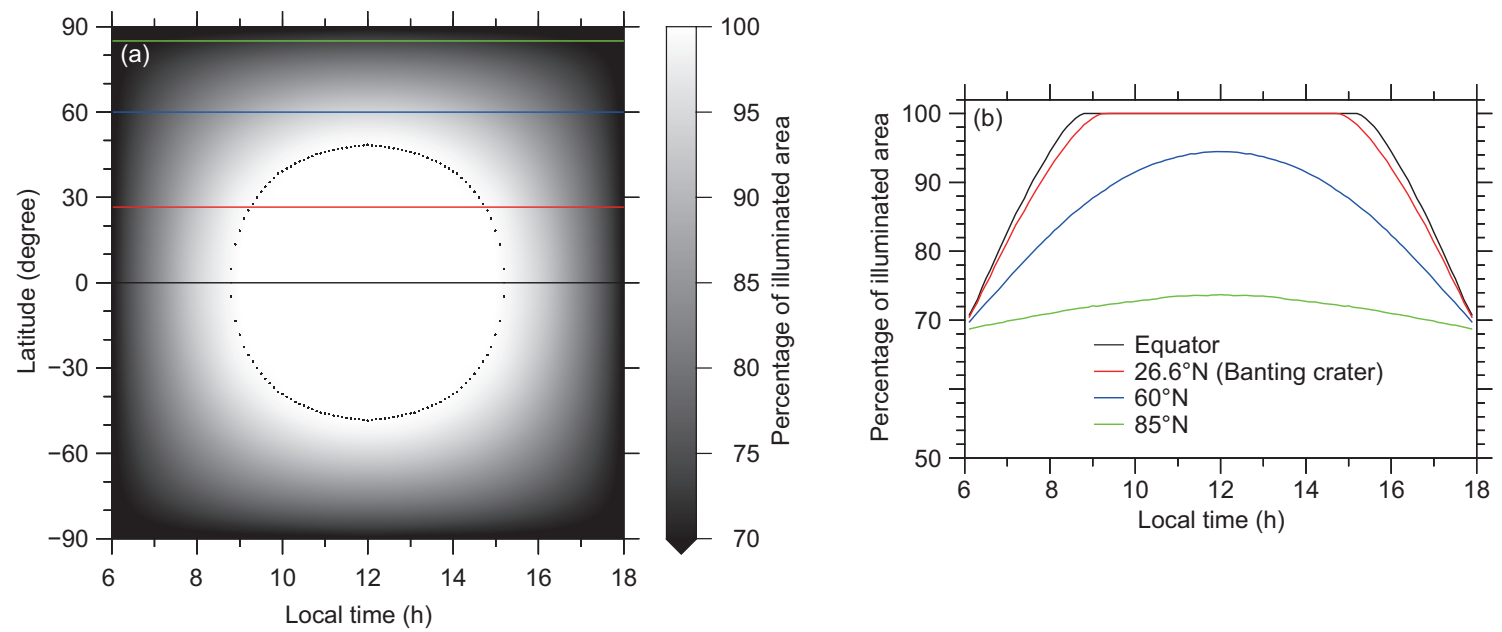

图 3 (网络版彩图)具有Banting陨坑特征的月球陨坑在(a)不同时间、不同位置的光照面积占比分布及(b)剖面分布. 注：由实 心点围成的圆圈区域表示陨坑的光照面积占比为 $100 \%$, 即没有任何自身遮蔽效应. $26.6^{\circ} \mathrm{N}$ 为Banting陨坑中心所在的纬度

Figure 3 (Color online) Distribution of percentage of illuminated area of craters at (a) different local times and locations and (b) their profiles. Note: the area enclosed by black dots indicates the craters are $100 \%$ illuminated, i.e., the craters are illuminated without self-shading effect. The center of Banting crater locates at $26.6^{\circ} \mathrm{N}$.

对比这4条剖面特征还可以发现: (1) 在黎明和 傍晚附近, 位于所有纬度陨坑的光照面积占比十分 接近; (2) 纬度越高陨坑的光照面积占比达到最大 值(黎明到正午)或降低到最小值(正午到傍晚)需要 的时间越长; (3) 纬度越高陨坑的光照面积占比变 化幅度就越小. 在将来的陨坑探测任务中, 由于低
纬度陨坑内太阳光照变化速率更快和幅度更大, 对 仪器或航天员的热冲击也将更强烈, 但光照面积 更大. 而在高纬度陨坑的探测中, 太阳光照变化速 率更慢、幅度更小, 由此给航天器或宇航员带来的 热冲击更小, 但是光照面积更小不利于太阳能的 利用. 

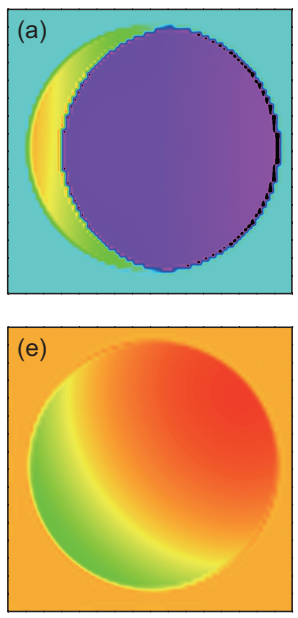
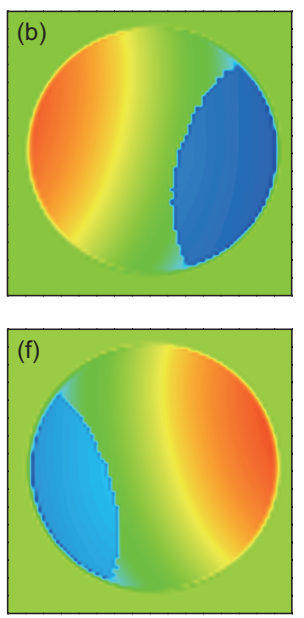
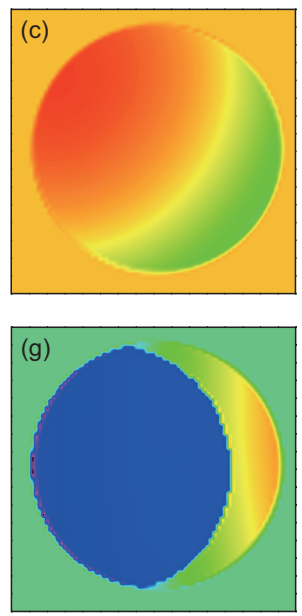
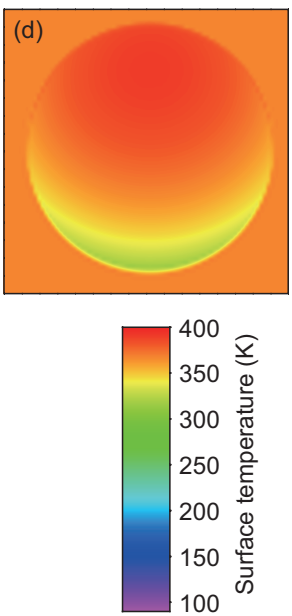

图 4 (网络版彩图)Banting陨坑 $\left(16.4^{\circ} \mathrm{E}, 26.6^{\circ} \mathrm{N}\right)$ 在不同地方时的温度分布. (a)-(g)对应的地方时分别为 06:30, 08:00, 10:00, 12:00, 14:00, 16:00和17:30. 注: 温度计算采用的参数为 $S=1366 \mathrm{~W} \mathrm{~m}^{-2}, A=0.127, \varepsilon=0.95^{\text {[32] }}$

Figure 4 Distribution of temperatures of Banting crater $\left(16.4^{\circ} \mathrm{E}, 26.6^{\circ} \mathrm{N}\right)$ at different local times. (a)- $(\mathrm{g})$ correspond to the local time $06: 30,08: 00$, 10:00, 12:00, 14:00, 16:00 and 17:30, respectively. Note: The parameters in our simulation are $S=1366 \mathrm{~W} \mathrm{~m}^{-2}, A=0.127, \varepsilon=0.95$ [32].

\section{2 陨坑温度分布}

基于陨坑的有效太阳辐照度分布和表面能 量平衡, 图4给出了Banting陨坑不同地方时的表面 温度分布. 为方便与有效太阳辐照度分布(图2)对 比, 模拟温度的时刻与其相对应. 可以看出, 太阳 辐照度高的坑壁具有较高的温度; 太阳辐照度低 和遮蔽区域的温度很低. 且越接近黎明(图4(a))或 傍晚(图4(g))时刻, 温度差异越大. 值得一提的是, 图4(a)和(g)所在的时刻距离日出和日落均相差 $30 \min$ (月球地方时), 但温度分布却并没有表现 出很好的对称性. 主要是因为黎明时刻的阴影区经 历了一个月球夜晚的热辐射损耗达到最低的 $98.6 \mathrm{~K}$; 从上文分析可知Banting陨坑从 $14: 42$ 才开始出现阴 影区, 因此坑内阴影区经历的最长热辐射损耗时间 为 $2.8 \mathrm{~h}$ (月球当地时), 温度最低仅为 $147.9 \mathrm{~K}$. 而光 照区的最高温度为 359.2 和 $366.7 \mathrm{~K}$, 二者相差并没有 阴影区那么大.

从时间范围来看, 越接近中午时刻(黎明到中 午和中午到傍晚)太阳辐射、坑壁反射和热辐射 作用越强. 结果如图4(b)和(f)所示, 光照区面积增 大, 阴影区面积减小, 且陨坑温度逐渐上升. 此 时二者光照区的最高温度分别为 384.0 和 $387.9 \mathrm{~K}$, 阴影区的最低温度分别为 144.4 和 $165.6 \mathrm{~K}$. 在 $10: 00$ (图4(c))和14:00(图4(e))时刻坑内光照面积占比 达 $100 \%$, 陨坑的自身遮蔽效应消失且表面温度主 要取决于有效太阳辐照度. 前者对应的最高温度 为 $395.3 \mathrm{~K}$, 最低温度为 $260.9 \mathrm{~K}$; 后者对应的最高温 度为 $396.3 \mathrm{~K}$, 最低温度为 $277.3 \mathrm{~K}$. 至此可以说明, 无 论是在上午还是下午陨坑光照区最高温度差别不 大, 但是阴影区或背光面的最低温度差异却很明显, 不过越临近中午这种差异均逐渐减小. 到了正午时 刻(图4(d)), 坑内向阳面最高温度为 $397.4 \mathrm{~K}$, 背光面 最低为 $321.8 \mathrm{~K}$. 此时陨坑接受太阳辐射能量最多, 温度趋向均匀分布且平均值也达到最大.

\section{3 可靠性验证}

以上模拟结果给出了 Banting陨坑温度随地方 时变化的特征, 为验证结果的可靠性本文进一 步将模拟结果与Diviner探测数据进行对比分析. 图5为覆盖研究区 $\left(16.333^{\circ} \mathrm{E}-16.523^{\circ} \mathrm{E}, 26.496^{\circ} \mathrm{N}-\right.$ $\left.26.666^{\circ} \mathrm{N}\right)$ 的表面温度随地方时的变化. 可以看出, Diviner的测量数据并未覆盖研究区的所有时段, 但在部分时间段数据分布比较集中. 为方便与模 拟结果进行比较, 且保证Banting陨坑数据的最大 覆盖范围, 本文分别选取07:21-07:36, 10:11-10:13, 12:24-12:27, 15:38-15:40,16:49-16:54时间范围内的 


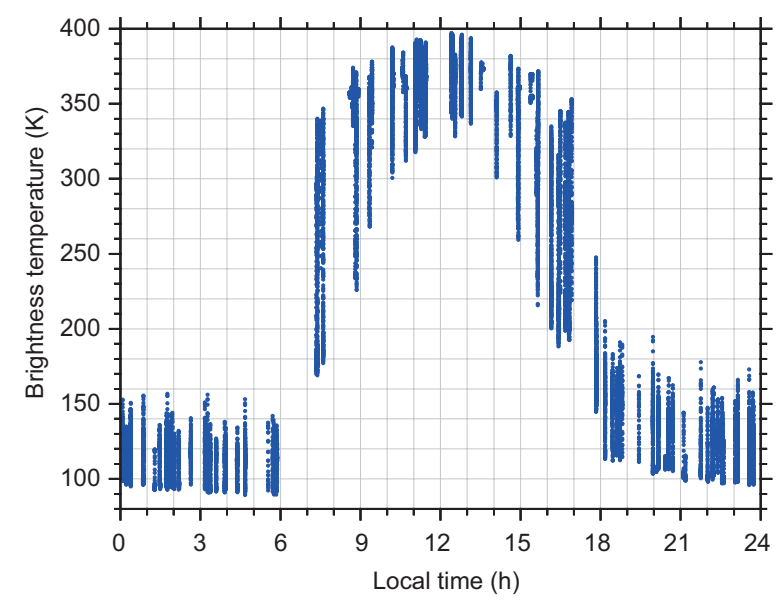

图 5 (网络版彩图)Banting陨坑 $\left(16.4^{\circ} \mathrm{E}, 26.6^{\circ} \mathrm{N}\right)$ 月表亮温随 地方时的变化

Figure 5 (Color online) Brightness temperature of Banting crater $\left(16.4^{\circ} \mathrm{E}, 26.6^{\circ} \mathrm{N}\right)$ versus local time.

Diviner数据分别表示07:30, 10:12，12:26，15:39 和16:52时刻的亮温. Vasavada等人 ${ }^{[24]}$ 通过分析覆 盖赤道地区的Diviner第7通道数据, 认为该通道的 光谱发射率 $\left(\varepsilon_{\mathrm{IR}}\right)$ 具有全球普适性, 其值为 0.98 . 则 表面温度可通过 $T_{\text {surf }}=T_{\mathrm{IR}} / \varepsilon_{\mathrm{IR}}{ }^{0.25}$ 计算得到, 其中 $T_{\mathrm{IR}}$ 为红外辐射亮温.

图6展示了Banting陨坑在不同地方时的温度分 布, 可以明显看到陨坑地形对温度分布的影响, 且 模拟结果和Diviner测量温度均表现出相似的温度 分布特征. 具体来看, 在07:30时刻坑内阴影区测量 的温度范围为169.1-346.8 K (图6(a)), 模拟温度范围 为 $157.4-377.6 \mathrm{~K}\left(\right.$ 图6 $\left.\left(\mathrm{a}^{\prime}\right)\right)$. 到了10:12(图6(b))坑内温 度迅速上升, 主要表现为向阳和背阳坡面的温度 差异. 坑内温度范围是300.5-387.6 K, 此时对应的 模拟温度范围是275.1-395.6 K (图6( $\left.\left.\mathrm{b}^{\prime}\right)\right)$. 而在接近 正午时刻陨坑的温度范围是341.6-399.2 K (图6(c)); 对应的模拟结果为320.7-397.4 K (图6( $\left.\mathrm{c}^{\prime}\right)$ ). 而到 了15:40, 坑内温度迅速下降且再次出现阴影区, 温 度范围是216.7-373.5 K (图6(d)); 对应的模拟温度 范围是197.1-390.0 K (图6(d')). 在16:52时刻, 坑内 测量温度范围是193.5-354.9 K (图6(e)), 模拟温度范 围是166.7-375.6 K (图6(e $\left.\mathrm{e}^{\prime}\right)$ ).

通过以上几个时刻的温度对比发现, 在向阳 面的模拟温度的最大值比Diviner测量的最大值普
遍偏大 $(12: 26$ 除外, 差值为-1.8 K), 范围在8-30.8 K; 在背光面或阴影区模拟温度的最小值比Diviner测 量的最小值普遍偏小, 范围在-26.8--11.7 K. 可能 有以下三点原因: (1) 由于没有实测数据, 模型中 给定的表面反照率和热红外发射率均是经验值, 而Banting陨坑表面的反照率可能比经验值更高. 这 会直接影响到陨坑光照区的温度并通过二次反射 和热辐射作用间接影响阴影区坑壁的温度, 下文将 具体讨论. 此外坑壁表面有大量石块, 且分布不均 一(图7), 使得坑壁表面月壤(0-10 cm深度)的热物理 性质更加复杂, 从而对表面温度变化产生重要的影 响 ${ }^{[23]}$. 而且这会使坑内太阳辐射的散射和热辐射变 得更复杂. 在本文的模型中均未考虑这些因素的影 响. (2) 选取的Diviner数据跨越一定的时间范围, 并 不是与模拟温度的时间完全一致. 比如, 从图 5 可以 看出, 即使在地方时很接近的如 16:00-17:00时间段 内, 最高或最低温度的差异可以达 $10 \mathrm{~K}$ 以上. 不过 需要注意的是在光照区和阴影区的交界处, 理论模 拟值表现出显著的温度突变. 而Diviner测量的温度 则表现出明显的渐变特征, 这主要是由于Diviner的 视场均获取了明暗交界处的热辐射信息得到了混合 值. (3) 坑壁的坡度变化与模型中采用的球面曲率存 在一定的差距, 导致计算的坑内热辐射散射场与实 际观测结果存在一定的偏差. 相对而言, 第一点原 因是主要因素, 也是在后续工作中需要考虑的关键 因素.

为进一步探讨反照率对陨坑内热辐射环境 的影响, 且考虑陨坑温度随时间变化的连续性, 选取坑底 $\left(16.4^{\circ} \mathrm{E}, 26.6^{\circ} \mathrm{N}\right)$ 作为研究对象, 计算得 到不同反照率条件下坑底温度随时间连续变 化的特征. 同时选取以坑底为中心、覆盖区域 为 $16.4^{\circ} \pm 0.01^{\circ} \mathrm{E}, 26.6^{\circ} \pm 0.01^{\circ} \mathrm{N}$ 的Diviner第 7 通道数 据, 从而得到Banting陨坑坑底白天测量温度与模 拟温度的对比, 如图8所示. 可以看到在13:00之 前, 模拟温度与观测结果具有非常相似的变化 趋势. 其中在 $07: 27$ 均表现出温度的急剧上升, 说 明坑底经历了从阴影区到光照区的快速转变. 而 在13:00之后二者也同样表现出随时间下降的趋势, 不过Diviner测量值变化更为迅速. 具体特征为观测 值温度在15:39急剧下降, 模拟温度则在16:38开始 

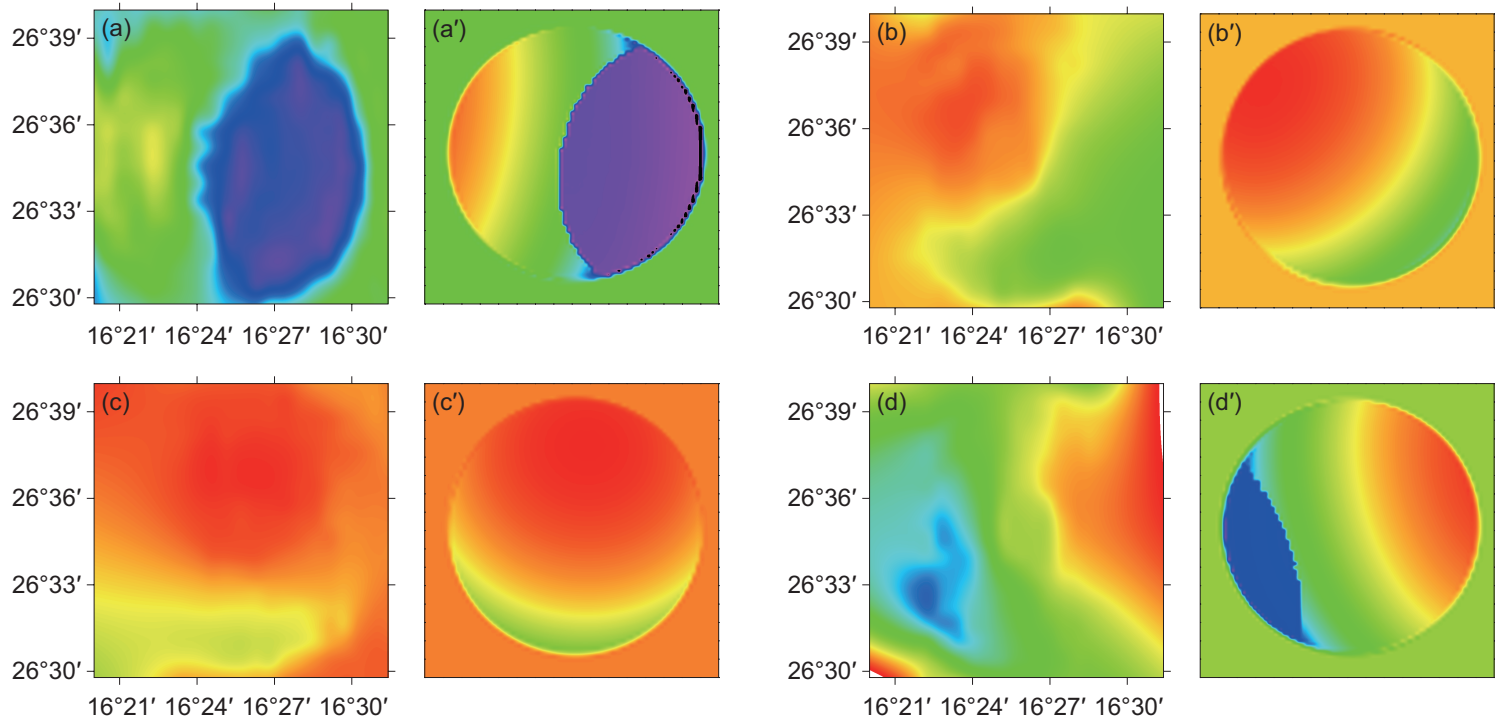

$16^{\circ} 21^{\prime} 16^{\circ} 24^{\prime} 16^{\circ} 27^{\prime} 16^{\circ} 30^{\prime}$
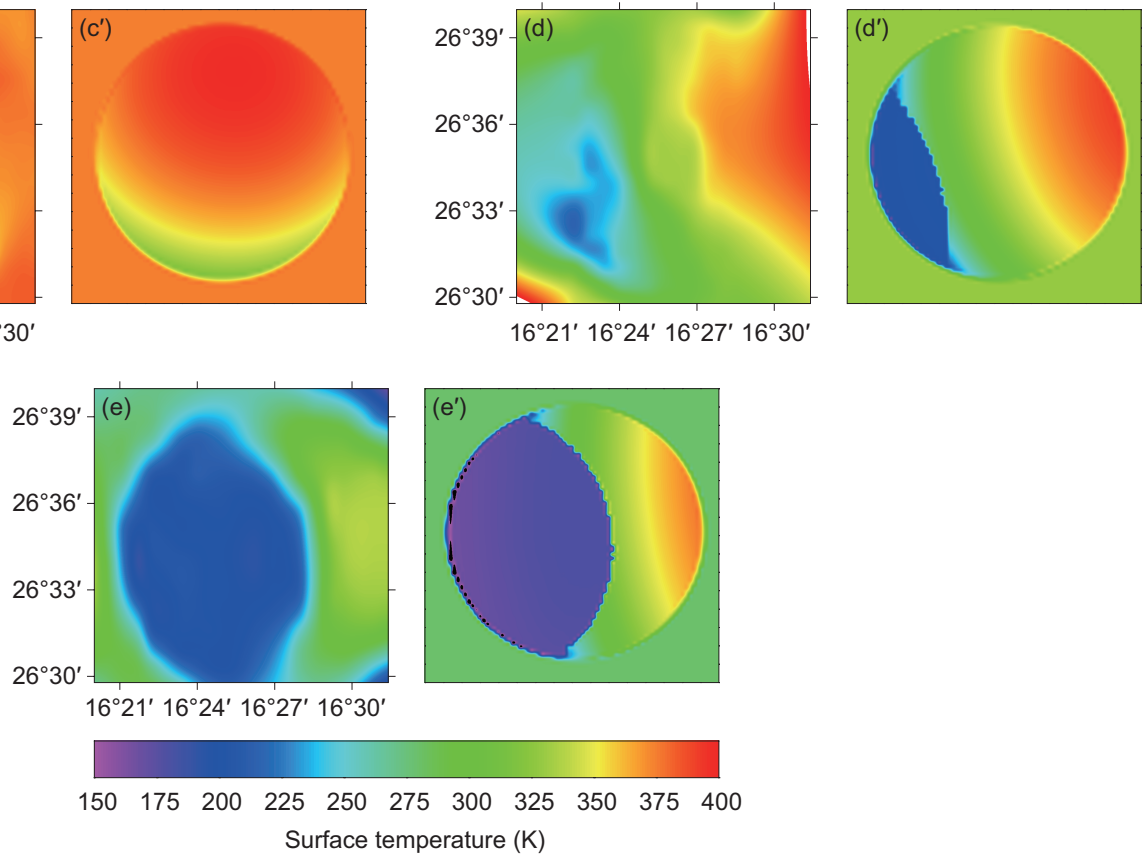

图 6 (网络版彩图)Banting陨坑 $\left(16.4^{\circ} \mathrm{E}, 26.6^{\circ} \mathrm{N}\right)$ 在不同地方时的Diviner测量温度(左列)与模拟温度(右列)的对比. (a)-(e)以 及 $\left(\mathrm{a}^{\prime}\right)-\left(\mathrm{e}^{\prime}\right)$ 对应的地方时分别为 $07: 30,10: 12,12: 26,15: 39$ 和16:52. 注: 温度计算采用的参数为 $S=1366 \mathrm{~W} \mathrm{~m}^{-2}, A=0.127$, $\varepsilon=0.95^{[32]}$, 红外发射率 $\varepsilon_{\mathrm{IR}}=0.98$, 且左列图中部分缺失数据的区域采取了插值填充

Figure 6 (Color online) Comparison of Diviner observations (left column) and simulated results (right column) for the temperatures at Banting crater $\left(16.4^{\circ} \mathrm{E}, 26.6^{\circ} \mathrm{N}\right)$ at different local times. (a)-(e)/( $\left.\mathrm{a}^{\prime}\right)-\left(\mathrm{e}^{\prime}\right)$ correspond to $07: 30,10: 12,12: 26,15: 39$ and 16:52, respectively. Note: the parameters in our simulation are $S=1366 \mathrm{~W} \mathrm{~m}^{-2}, A=0.127, \varepsilon=0.95$ [32], and $\varepsilon_{\mathrm{IR}}=0.98$, and the missing data in left column are interpolated.

急剧下降. 从整体时间范围来看, Banting陨坑坑底 的模拟温度以12:00为界呈对称分布, 而Diviner测量 温度分布则更加复杂. 可能是坑内石块堆积物等因 素使坑底的热辐射环境变得更加复杂导致的.

反照率对光照区温度的影响非常大, 随着反照 率增大月表温度快速降低. 例如, 在正午时刻, 当 反照率从 0.1 升高到 0.127 变化时, 表面温度降低约 $2.7 \mathrm{~K}$. 当反照率从 0.127 增加到 0.2 时, 温度降低约 $7.5 \mathrm{~K}$. 反照率继续增加到 0.3 时, 这时温度迅速降低 约 $11.4 \mathrm{~K}$. 而当坑底处于阴影区时, 反照率变化对其 温度的影响不如处于光照区时那么明显, 但仍表现
出随着反照率增加温度降低的趋势. 需要说明的 是, 虽然反照率升高增加了阴影区接受太阳散射光 的能量, 但其阴影区对入射散射光的反照率也同样 增加. 最后的作用结果是随反照率增加温度逐渐降 低. 综合对比来看, 本文陨坑温度计算中选取的反 照率 0.127 具有一定合理性.

通过上述分析发现, 即使给定反照率较大的取 值范围(0.1-0.3), 模拟温度与Diviner观测结果仍然 存在一定的偏差. 由此可见, 除了反照率, 石块含量 及分布也是控制陨坑温度的重要因素. 在后续工作 中我们将进一步结合反照率和石块含量及分布对温 


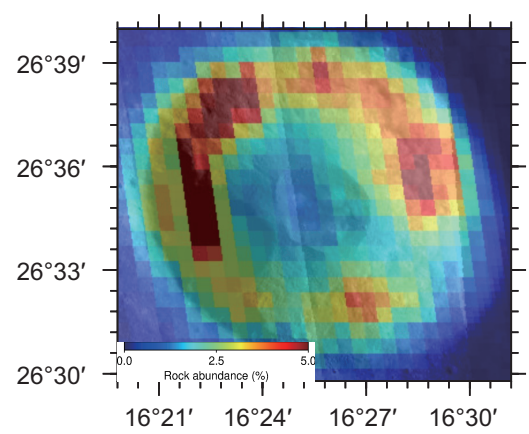

图 7 (网 络版彩图) Banting陨 坑 $\left(16.4^{\circ} \mathrm{E}, 26.6^{\circ} \mathrm{N}\right)$ 表 面石块分布. 数据来源于http://ode.rsl.wustl.edu/moon/ indexProductSearch.aspx, 底图为LRO WAC光学影像

Figure 7 (Color online) Distribution of rock abundance in Banting crater $\left(16.4^{\circ} \mathrm{E}, 26.6^{\circ} \mathrm{N}\right)$. The data are from http://ode.rsl.wustl.edu/moon/ indexProductSearch.aspx, and the base map is LRO WAC image.

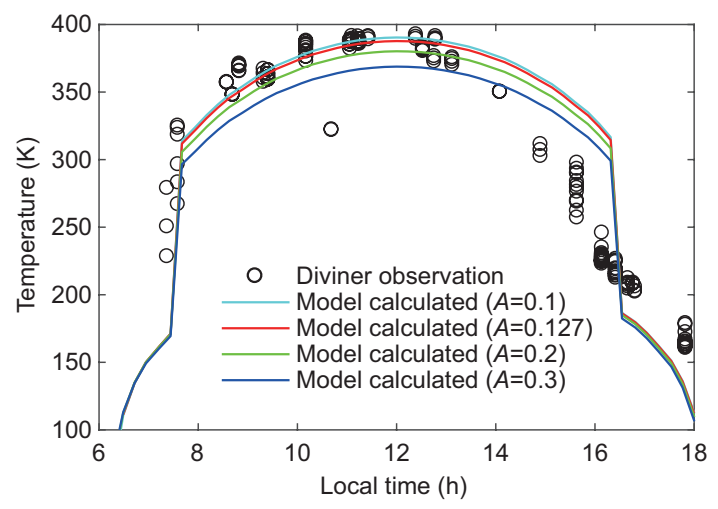

图 8 (网络版彩图) Banting陨坑坑底中心 $\left(16.4^{\circ} \mathrm{E}, 26.6^{\circ} \mathrm{N}\right)$ 不 同反照率条件下的模拟温度与Diviner 测量温度对比. $\varepsilon_{\mathrm{IR}}=0.98$. 注: Diviner第7通道数据覆盖范围为 $\left(16.4^{\circ} \pm 0.01^{\circ}\right) \mathrm{E}$, $\left(26.6^{\circ} \pm 0.01^{\circ}\right) \mathrm{N}$

Figure 8 (Color online) Comparison of temperature at the center of Banting crater $\left(16.4^{\circ} \mathrm{E}, 26.6^{\circ} \mathrm{N}\right)$ between Diviner measurements and albedo dependent simulation results. $\varepsilon_{\mathrm{IR}}=0.98$. Note: The coverage of channel 7 data of Diviner is $\left(16.4^{\circ} \pm 0.01^{\circ}\right) \mathrm{E},\left(26.6^{\circ} \pm 0.01^{\circ}\right) \mathrm{N}$.

度的影响, 探讨月表不同时空条件下陨坑的热辐射 环境特征. 为将来陨坑探测任务中区域选址、太阳 能资源利用等提供一定的参考依据.

\section{4 结论}

太阳辐射是驱动月表热环境变化的主要因素, 而广泛分布的陨坑使得月表温度分布变得更加复 杂. 本文以Banting简单陨坑为例, 首先基于LOLA数
据并通过数值方法构造了Banting陨坑的三维模型. 接着计算了陨坑在白天不同时刻的有效太阳辐照 度分布, 发现越接近黎明或傍晚时刻陨坑的自身 遮蔽效应越显著, 太阳辐射对陨坑的影响也就越 弱. 并进一步假设月表任意位置均具有Banting陨 坑特征的陨坑分布, 计算了白天任意时刻陨坑的 光照面积占比分布. 结果表明, 在 $48.2^{\circ} \mathrm{N} / \mathrm{S}$ 范围且当 地时间在08:48-15:12之间的陨坑, 光照面积占比达 到 $100 \%$, 即在此范围内陨坑没有自身遮蔽效应. 而 在靠近黎明/傍晚时刻或两极位置的陨坑的自身遮 蔽效十分明显, 光照面积占比最低降到 $70 \%$ 以下.

基于陨坑的有效太阳辐照度分布和表面能量平 衡, 本文计算了Banting陨坑在不同时刻的温度分布. 结果表明, 即使在黎明或傍晚向阳坑壁可以接受较 多的太阳辐射能量, 与阴影区表现出很大的温度差 异. 越接近正午时刻, 光照区面积增加温度升高, 阴 影区面积减小温度也升高. 在整个月球白天, 光照 区的最高温度随时间变化幅度较小, 而阴影区或背 光面的最低温度随时间变化的幅度较大.

为验证模拟结果的可靠性, 本文进一步选取 了覆盖Banting陨坑的Diviner第7通道亮温数据, 分 别与 $07: 30,10: 12,12: 26,15: 39$ 和 $16: 52$ 时刻的模拟温 度进行对比分析. 结果表明模拟温度与Diviner测 量温度均表现出相似的温度分布特征. 在向阳面 的模拟温度的最大值比测量温度的最大值普遍偏 大(12:26除外, 差值为-1.8 K), 范围在8-30.8 K; 在背 光面或阴影区模拟温度的最小值比测量温度的最 小值普遍偏小, 范围在-26.8--11.7 K. 这些可能是 由于模型中表面反照率、发射率以及陨坑坡面堆 积物所致. 再通过对比Banting陨坑坑底的模拟温度 与Diviner观测温度, 发现模拟结果与观测值表现出 相似的变化趋势. 在07:27均表现出温度的急剧上 升, 而在13:00之后二者也同样表现出随时间下降的 趋势, 不过Diviner测量值变化更为迅速. 从整体时间 范围看, 模拟温度与Diviner观测结果的一致性验证 了模拟结果的可靠性. 在未来工作中, 我们将进一 步考虑不同深径比对陨坑表面温度的影响, 同时结 合月球星历数据建立月球陨坑温度变化的实时模 型, 为将来月球陨坑探测和月表活动提供一定的参 考依据. 
1 Li X Y, Wang S J, Cheng A Y. A review of lunar-surface temperature model (in Chinese). Adv Earth Sci, 2007, 22: 480-485 [李雄耀, 王世杰, 程 安云. 月球表面温度物理模型研究现状. 地球科学进展, 2007, 22: 480-485]

2 Li X, Wang S, Zheng Y, et al. Estimation of solar illumination on the Moon: A theoretical model. Planet Space Sci, 2008, 56: 947-950

3 Feng X, Guo Q. The lunar surface temperature real-time model. J Remote Sensing, 2017

4 Liu S H. Lunar surface solar irradiance simulation model based on computational geometry (in Chinese). Spacecraft Eng, 2014, 23: 19-26 [刘书 豪. 应用计算几何的月面太阳辐照度仿真模型. 航天器工程, 2014, 23: 19-26]

5 Meng Z G, Xu Y, Cai Z C, et al. Influence of lunar topography on simulated surface temperature. Adv Space Res, 2014, 54: 2131-2139

6 Zhang J D, Meng Z G, Ping J S, et al. Preliminary study of illumination characteristics of aristarchus plateau using LOLA data (in Chinese). J Deep Space Explor, 2017, 4: 171-177 [张吉栋, 孟治国, 平劲松, 等. 基于LOLA数据的Aristarchus高原光照特性初步研究. 深空探测学报, 2017, 4: 171-177]

7 Zhang J D, Meng Z G, Zhu Y Z, et al. Research on solar radiation of Von Kármán Crater using LOLA data (in Chinese). J Deep Space Explor, 2018, 5: 12-19 [张吉栋, 孟治国, 朱蕴哲, 等. 基于LOLA 数据的冯.卡门撞击坑太阳辐射研究. 深空探测学报, 2018, 5: 12-19]

8 Colaprete A, Schultz P, Heldmann J, et al. Detection of water in the LCROSS ejecta Plume. Science, 2010, 330: 463-468

9 Rubanenko L, Aharonson O. Stability of ice on the Moon with rough topography. Icarus, 2017, 296: 99-109

10 Vasavada A. Near-surface temperatures on mercury and the Moon and the stability of polar ice deposits. Icarus, 1999, 141: 179-193

11 Noda H, Araki H, Goossens S, et al. Illumination conditions at the lunar polar regions by KAGUYA (SELENE) laser altimeter. Geophys Res Lett, 2008, 35: L24203

12 Bussey D B J, McGover n J A, Spudis P D, et al. Illumination conditions of the south pole of the Moon derived using Kaguya topography. Icarus, 2010, 208: 558-564

13 Paige D A, Siegler M A, Zhang J A, et al. Diviner Lunar Radiometer observations of cold traps in the Moon's south polar region. Science, 2010, 330: 479-482

14 Mazarico E, Neumann G A, Smith D E, et al. Illumination conditions of the lunar polar regions using LOLA topography. Icarus, 2011, 211: $1066-1081$

15 Hao W F, Li F, Yan J G, et al. Lunar polar illumination based Chang'E-1 laser altimeter (in Chinese). Chin J Geophys, 2012, 55: 46-54 [郝卫峰, 李斐, 漂建国, 等. 基于“嫦娥一号”激光测高数据的月球极区光照条件研究. 地球物理学报, 2012, 55: 46-52]

16 Pettit E, Nicholson S B. Lunar radiation and temperatures. Astrophys J, 1930, 71: 102-135

17 Sinton W M. CHAPTER 11-Temperatures on the Lunar Surface. Heidelberg: Elsevier Inc., 1961

18 Buhl D, Welch W J, Rea D G. Reradiation and thermal emission from illuminated craters on the lunar surface. J Geophys Res Planets, 1968, 73: 5281-5295

19 Davidsson B J R, Rickman H. Surface roughness and three-dimensional heat conduction in thermophysical models. Icarus, 2014, 243: 58-77

20 Paige D A, Foote M C, Greenhagen B T, et al. The lunar reconnaissance orbiter diviner lunar radiometer experiment. Space Sci Rev, 2010, 150: $125-160$

21 Hayne P O, Bandfield J L, Siegler M A, et al. Global regolith thermophysical properties of the moon from the diviner lunar radiometer experiment. J Geophys Res Planets, 2017, 122: 2371-2400

22 Williams J P, Paige D A, Greenhagen B T, et al. The global surface temperatures of the Moon as measured by the Diviner Lunar Radiometer Experiment. Icarus, 2017, 283: 300-325

23 Bandfield J L, Ghent R R, Vasavada A R, et al. Lunar surface rock abundance and regolith fines temperatures derived from LRO Diviner Radiometer data. J Geophys Res Planets, 2011, 116: E00H02

24 Vasavada A R, Bandfield J L, Greenhagen B T, et al. Lunar equatorial surface temperatures and regolith properties from the Diviner Lunar Radiometer Experiment. J Geophys Res Planets, 2012, 117: E00H18

25 Chen S, Meng Z G, Zhang J D, et al. Research on microwave radiation characteristics at Tycho crater area (in Chinese). Sci Sin-Phys Mech Astron, 2016, 46: 029608 [陈思, 孟治国, 张吉栋, 等. Tycho 撞击坑地区微波热辐射特性研究. 中国科学: 物理学 力学 天文学, 2016, 46: 029608]

26 Sun L Z, Ling Z C, Zhang J, et al. The spectral characteristics and remote detection of lunar Mg-spinel: A case study of Tycho Crater (in Chinese). Sci Sin-Phys Mech Astron, 2016, 46: 029607 [孙灵芝, 凌宗成, 张江, 等. 月表镁尖晶石矿物的光谱特征与遥感探测: 以第谷撞击坑为例. 中 国科学: 物理学 力学 天文学, 2016, 46: 029607]

27 Pike R J. Size-dependence in the shape of fresh impact craters on the moon. In: Impact and Explosion Cratering: Planetary and Terrestrial Implications, Flagstaff, 1977. 489-509 
Ingersoll A P, Svitek T, Murray B C. Stability of polar frosts in spherical bowl-shaped craters on the moon, Mercury, and Mars. Icarus, 1992, 100: $40-47$

29 Stopar J D, Hawke B R, Robinson M S, et al. Distribution, occurrence, and degradation of impact melt associated with small lunar craters. In: 43rd Lunar and Planetary Science Conference. The Woodlands, 2012. 115-118

30 Davidsson B J R, Rickman H, Bandfield J L, et al. Interpretation of thermal emission. I. The effect of roughness for spatially resolved atmosphereless bodies. Icarus, 2015, 252: 1-21

31 Aharonson O, Schorghofer N. Subsurface ice on Mars with rough topography. J Geophys Res Planets, 2006, 111: E11007

32 Racca G D. Moon surface thermal characteristics for moon orbiting spacecraft thermal analysis. Planet Space Sci, 1995, 43: 835-842

\title{
Numerical simulation of effective solar irradiance and temperatures at simple crater of lunar dayside
}

\author{
GAN Hong ${ }^{1,2 *}$, LI XiongYao ${ }^{3,4} \&$ WEI GuangFei ${ }^{2,3,4}$ \\ ${ }^{1}$ Analyzing and Testing Center, Guizhou Institute of Technology, Guiyang 550003, China; \\ ${ }^{2}$ State Key Laboratory of Lunar and Planetary Sciences, Macau University of Science and Technology, Macau, China; \\ ${ }^{3}$ Center for Lunar and Planetary Sciences, Institute of Geochemistry, Chinese Academy of Sciences, Guiyang 550081, China; \\ ${ }^{4}$ Center for Excellence in Comparative Planetology, Chinese Academy of Sciences, Hefei 230026, China
}

Solar radiation plays an important part in lunar surface thermal environment. The topographic effect was usually ignored in previous work, which might cause great uncertainties in surface temperature simulation. To better understand the surface temperatures of the craters at any time and on any location of the Moon, we simulated the effective solar irradiance and temperatures of the Banting crater as an example. In this work, we firstly constructed a three-dimensional Banting crater $\left(16.4^{\circ} \mathrm{E}, 26.6^{\circ} \mathrm{N}\right)$ model based on the elevation data obtained from the Lunar Orbiter Laser Altimeter onboard Lunar Reconnaissance Orbiter (LRO), and then calculated the distribution of the effective solar irradiance at different local times. In addition, we calculated the distribution of percentage of illuminated area of the Banting-like craters at any day time and on all locations of the Moon. These results show that the craters located within $48.2^{\circ} \mathrm{N} / \mathrm{S}$ and at the time of $08: 48-15: 12$ are illuminated without any self-shading, while that located at high latitudes or near to dawn/dusk endure different degrees of the self-shading effect. Finally, we simulated the temperatures of the Banting crater at different local times based on the surface radiation balance. Compared to the temperatures measured by Diviner aboard LRO, the results show that the simulated temperatures are well consistent with the Diviner observations, which verified our models and simulation results.

\section{Moon, simple crater, solar irradiance, temperature, numerical simulation}

PACS: $95.30 . J x, 95.85 . H p, 96.20 .-n, 96.35 . G t$

doi: 10.1360/SSPMA2018-00348 